\title{
Vaccination and Host Marek's Disease-Resistance Genotype Significantly Reduce Oncogenic Gallid alphaherpesvirus 2 Telomere Integration in Host Birds
}

\author{
Marla C. McPherson ${ }^{a, b}$ Hans H. Cheng ${ }^{c}$ Justin M. Smith ${ }^{a}$ Mary E. Delany ${ }^{a}$ \\ ${ }^{a}$ Department of Animal Science, University of California, Davis, CA, ${ }^{b}$ Department of Surgery, Stanford University \\ School of Medicine, Stanford, CA, and 'USDA, ARS, Avian Disease and Oncology Laboratory, East Lansing, MI, USA
}

\section{Keywords}

Chicken genome · FISH · Genetic resistance · Herpesvirus ·

Marek's disease $\cdot$ Rispens vaccine $\cdot$ Telomere integration

\begin{abstract}
Marek's disease (MD) is an infectious disease characterized by lymphomas and high mortality in susceptible chickens. The causative and ubiquitous alpha-herpesvirus known as $M D$ virus (MDV) integrates into host telomeres during early infection through latency, known to be an important phase for oncogenic transformation. Herein, we sought to determine the influence of vaccination and host genetics on the temporal dynamics of MDV-host genome interactions. We studied integration profiles using $2 \mathrm{MD}$ vaccines that vary in protective efficacy in 2 genetic lines that differ in MD resistance/susceptibility. Virus integration of both oncogenic MDV and vaccine strains was observed in both MD susceptible and resistant birds, however, the lines differed in their dynamic telomere-integration profiles. Notably, the resistant host genotype exhibited a smaller percentage of replicating cells with the virus telomere-integrated only phenotype as compared to the susceptible genotype. Vaccination with Rispens, the most protective MD vaccine, also reduced the establishment of the virus telomere-integrated only
\end{abstract}

\section{KARGER}

E-Mail karger@karger.com www.karger.com/cgr

\section{The Author(s) \\ Published by S. Karger AG, Basel \\ Karger
Open access}

This article is licensed under the Creative Commons AttributionNonCommercial-NoDerivatives 4.0 International License (CC BYNC-ND) (http://www.karger.com/Services/OpenAccessLicense) Usage and distribution for commercial purposes as well as any distribution of modified material requires written permission. phenotype, suggesting a significant role of the phenotype in MD lymphoma development. The effect of Rispens vaccination was most dramatic in the susceptible genotype. These results suggest important connections between vaccinal immunity, MDV telomere integration, virus-induced oncogenesis, and virus-host genome interactions in the context of host genetics and disease susceptibility.

\section{(C) 2018 The Author(s)}

Published by S. Karger AG, Basel

Marek's disease (MD) is a global threat to poultry production and is characterized by lymphoma development in visceral organs and nerves, resulting in paralysis and death in susceptible chickens. To limit disease incidence in commercial flocks, the industry employs several strategies including selective breeding for enhanced disease resistance and widespread vaccination. MD virus (MDV), a ubiquitous Gallid alphaherpesvirus [Churchill and Biggs, 1967, 1968], is the known causal pathogen of this disease and remains a challenge to mitigate given repeated viral evolution to evade widespread vaccinal control. MDV integrates into host telomeres of mitotically dividing cells [Delecluse and Hammerschmidt, 1993; Robinson et al., 2010, 2014], as early as 1 day post challenge (dpc), through a mechanism facilitated by MDV telomer- 
ic repeats [Kaufer et al., 2011; Greco et al., 2014]. In the MDV infection cycle, oncogenic strains transition from a cytolytic replication stage, during which the viral genome takes an episomal and extrachromosomal form, to a latent stage, characterized by evasion of host immune responses [Biggs, 1968; Adldinger and Calnek, 1973; Baigent and Davison, 2004; Osterrieder et al., 2006; Arumugaswami et al., 2009; Gimeno et al., 2011] with the latter having a temporal overlap with MDV-host telomere integration events [Robinson et al., 2010, 2014] and decline in the quantity of extrachromosomal MDV genomes [Kaschka-Dierich et al., 1979; Delecluse and Hammerschmidt, 1993]. Latently infected host CD4 T lymphocytes are the target cell population for transformation events and subsequent lymphoma development, around 2-3 weeks after infection in susceptible birds [Witter, 1984; Calnek, 2001; Burgess and Davison, 2002; Nair, 2005; Trapp et al., 2006].

Host bird genetics contributes to MD resistance/susceptibility as well as the effectiveness of the MD vaccines [Bacon and Witter, 1994; Sarson et al., 2008; Chang et al., 2010, 2012]. The chicken major histocompatibility complex (MHC) has long been known to have a major influence on MD disease incidence [Briles et al., 1983; Bacon, 1987; Schat et al., 1994; Bacon et al., 2000; Sarson et al., 2008; Miller and Taylor, 2016]. Conveniently, blood group and DNA markers have enabled poultry breeding companies to select birds with superior MD resistance. In addition to the $\mathrm{MHC}$, other genetic factors exert a major influence on MD resistance. For example, Avian Disease and Oncology Laboratory (ADOL) experimental inbred lines $6_{3}$ and $7_{2}$ chickens share the same MHC haplotype, yet differ greatly in resistance to MD. As these non-MHC loci are smaller in genetic effect, identifying them has been more challenging though recent efforts based on allele-specific expression in response to MDV challenge have been able to identify a large collection that accounts for over $80 \%$ of the genetic variance [Cheng et al., 2015].

Since the early 1970s, MD control has relied heavily on mass vaccination of flocks [Calnek, 2001]. MD vaccination strategies commonly consist of monovalent injection of herpesvirus of turkey (HVT, apathogenic MDV serotype 3) or polyvalent injections of HVT [Churchill et al., 1969; Okazaki et al., 1970; Kingham et al., 2001], SB-1 (apathogenic MDV serotype 2) [Schat and Calnek, 1978; Spatz and Schat, 2011], or Rispens/CV1988 (attenuated MDV serotype 1) [Rispens et al., 1972; Calnek et al., 1983; Petherbridge et al., 2003; Spatz et al., 2007]. The MD vaccines protect birds against disease progression and lymphoma development induced with oncogenic strains

Vaccination and Host Genetics Impact MDV-Host Genome Interaction Profiles
[Chang et al., 2010; Haq et al., 2010] through non-sterilizing immunity [Nair, 2005] in both susceptible and resistant host birds. The genomes of these strains are highly similar to the oncogenic strains, but crucial differences exist [McPherson and Delany, 2016]. The MD vaccine viruses replicate in host cells and transmit to other birds [Baigent et al., 2005; Islam et al., 2007; Tan et al., 2007; Gimeno et al., 2011; Islam et al., 2013; McPherson and Delany, 2016; McPherson et al., 2016], but do not induce disease or lymphomas [Calnek and Witter, 1997]. Despite improved understanding of vaccine-related immunity [Witter, 1984; Osterrieder et al., 2006], an explanation for the anti-tumor effect of vaccination has not yet been established. Vaccine protective efficacy can vary with the vaccine serotype, host MHC haplotype, and other aspects of the host genetics [Bacon, 1987; Bacon and Witter, 1992, 1993, 1994; Chang et al., 2010, 2012, 2014]. Rispens provides the most effective protection against the most virulent MDV strains in susceptible birds [Haq et al., 2012; Chang et al., 2014].

The MD vaccine strains have been detected in a latent form in vivo [Calnek and Witter, 1997], although infrequently and highly delayed as compared to oncogenic $\mathrm{MDV}$, and are capable of host telomere integration [McPherson et al., 2016], partially resembling oncogenic MDV. Nevertheless, the vaccines do not result in a transformation-related phenotype, known as MDV telomereintegrated only, in dividing host cells [Robinson et al., 2010, 2014; McPherson et al., 2016]. Noteworthy, a similar pattern of host chromosome association and integration [Robinson et al., 2014] is observed with the Meq-deleted ( $\Delta \mathrm{Meq}$ ) $\mathrm{MDV}$, a recombinant MDV that lacks the viral oncogene and is a candidate MD vaccine [Silva et al., 2010].

Host genetic background (MHC and non-MHC) contributes to the effectiveness and behavior of the MD vaccine strains, which has been investigated for lines $6_{3}$ (resistant) and $7_{2}$ (susceptible) [Bacon and Witter, 1994; Chang et al., 2010, 2012]. In general, the serotype 1 vaccines, particularly Rispens, are more effective compared to serotype 2 vaccines. The bivalent serotype $2+3$ vaccine is also more protective than serotype $3 \mathrm{HVT}$ alone [Bacon and Witter, 1994]. The impact of host genotype on MD vaccine strain replication and shedding, and other aspects of the viral cycle within host lymphoid cells are poorly understood.

Our central hypothesis for this research was that oncogenic MDV and MD vaccine strain interactions with the host genome are influenced by the host genotype and contribute to the outcome of MD pathogenesis. Furthermore, we hypothesize that vaccination with Rispens MD 
vaccine influences oncogenic MDV interactions with the host genome in challenged birds in either an MD-resistant or -susceptible genetic background. Here, we tested these hypotheses by investigating chromosomal association and integration profiles of oncogenic MDV and MD vaccine viruses in inbred chicken disease genotypes $6_{3}$ (resistant) versus $7_{2}$ (susceptible) via previously defined cytogenomic methods. Our results contribute to a better understanding of the impact of host genotype and vaccination on MDV interactions with the host genome in dividing cells during early infection. Our findings also enlighten how the MD vaccines may work to reduce the oncogenic effect of virulent MDV strains.

\section{Materials and Methods}

Chickens, MDV and MD Vaccines, and Procedures

Experimental White Leghorn birds were from highly inbred lines $\sigma_{3}$ (resistant to MD-lymphoma development, also known as "resistant") or $7_{2}$ (susceptible to MD-lymphoma development and related mortality, also known as "susceptible") from the USDA ADOL [Sharma and Stone, 1972; Stone, 1975], cared for under approved animal care protocols. All institutional and national guidelines for the appropriate care and use of laboratory animals were followed. For the single-treatment group, i.e., viral challenge or vaccination only, day-of-hatch chicks from both lines were intraabdominally injected with 2,000 pfu Rispens/CVI988 (hereafter referred to as "Rispens") only, 2,000 pfu HVT only, 2,000 pfu Md5 strain MDV only, or received a mock inoculation. For the second group, i.e., dual treatment of vaccination and viral challenge, dayof-hatch chicks from both genotypes were intra-abdominally vaccinated with 2,000 pfu of Rispens or mock vaccinate (saline injection), then all birds were challenged with 2,000 pfu of Md5 virulent MDV at 4 days post hatch (dph). Chicks were hatched and maintained in separate Horsfall-Bauer isolation chambers at ADOL.

\section{Tissue Collection and Processing}

Mitotic chromosome preparations were harvested from 4 splenic-derived samples (1 sample per bird) collected at 1, 4, 7, 14, and 21 days after viral challenge or vaccination (dpc, dpv) for single-treatment birds and at 1, 4, 7, 14, and $21 \mathrm{dpc}$ for dual-treatment birds; thus, birds in the latter groups were older at the time of tissue sampling (i.e., 5, 8, 11, 18, and $25 \mathrm{dph}$ ). At least 3 tissues were processed per treatment as outlined previously [McPherson et al., 2016].

\section{MDV-Specific FISH}

Slide hybridization was carried out as previously described [Delany et al., 2007], with adaptations for labeling MDV as detailed in McPherson et al. [2016]. Image capture occurred within $24 \mathrm{~h}$ of MDV FISH and DAPI counterstaining.

\section{Cytogenetic Analysis}

Metaphase and interphase cell images were collected using an Olympus BX41 epifluorescence microscope equipped with an automatic filter wheel (Chroma Technology 82000, DAPI/FITC/
TRITC filter set), X-Cite 120 Series metal-halide fiber optic lamp, and Applied Imaging software (CytoVision 7.4 GENUS, Leica Biosystems). DAPI (host chicken DNA) and FITC (MDV DNA) exposure times were consistent across slide cell image captures. From 20 to 80 metaphase (dividing) cell images (average of 50) were captured and analyzed per individual sample or bird in each FISH experiment. Negative control (no treatment) samples were incorporated in all MDV FISH experiments to ensure that the MDV genome-containing $\mathrm{BAC}$ probe was hybridizing specifically to the MDV genome, as indicated by the absence of FITC signals from all terminal and interstitial telomeres, as described [Robinson et al., 2010]. All captured mitotic metaphase cells were categorized as 1 of 4 cytogenomic host-virus phenotypes as previously described [Robinson et al., 2014; McPherson and Delany, 2016] and are depicted in Figure 1: null (no signals detected); chromosome-associated (dispersed signals over and around the chromosomes); chromosome-associated/telomere-integrated (associated signals along with distinct, punctate and bright signal(s) at the telomeres); or telomere-integrated only (only the distinct telomeric signals detected).

For the Rispens-vaccinated/Md5-challenged (dual treatment) birds in the second set of experiments, mitotic cells with the telomere-integrated only virus-host phenotype represent solely the oncogenic MDV (Md5) infection, due to previous results and data here within demonstrating that the MD vaccines alone do not, or very rarely, exhibit the telomere-integrated only phenotype in dividing host splenic cell populations [McPherson et al., 2016]. However, the chromosome-associated phenotype or the chromosome-associated/telomere-integrated phenotype represent host mitotic cells containing the Rispens vaccine strain, the oncogenic Md5 strain, or both [McPherson et al., 2016].

\section{Statistical Analysis}

Raw cell counts for each of the 4 cytogenomic viral phenotypes were collected by analyses of mitotically dividing cell populations from 3 individual bird samples within each genotype $\left(6_{3}\right.$ and $\left.7_{2}\right)$, treatment group (Md5 oncogenic MDV challenge, Rispens or HVT MD vaccination), and timepoint $(1,4,7,14,21 \mathrm{dpc} / \mathrm{dpv})$. The raw count data were transformed using a logarithm of the odds (logit) transformation [logit $(k / n)=\ln \{k+1 / n-k+1\}]$, in which $k$ out of $n$ total analyzed cells were observed for any given viral phenotype within a bird, treatment group, and timepoint, as described [Robinson et al., 2014]. The logit transformation is applied to fit categorical data in the form of proportions to a linear model. The transformed data were evaluated by two-factor ANOVA and Tukey's multiple comparisons of means post-hoc test, with a $95 \%$ family-wise confidence interval, in $\mathrm{R}$ (version 3.3.1) [R Development Core Team, 2016] to establish statistically significant differences within the genotypes. The count of metaphase cells identified within each viral phenotype was the dependent variable in ANOVA analyses. The Tukey's method of multiple comparison analysis tests all pairwise differences between groups and reduces the probability of type I error. The effects of MDV strain (treatment type) and dpc/dpv (timepoint) on viral phenotype within a given chicken genotype were calculated by this method. Furthermore, statistically significant differences in the normalized phenotype data between genotypes (for each treatment and timepoint) were determined by multiple one-way ANOVA analyses in R.

The data from the dual-treatment bird groups were collected, logit-transformed, and evaluated by two-factor ANOVA and 


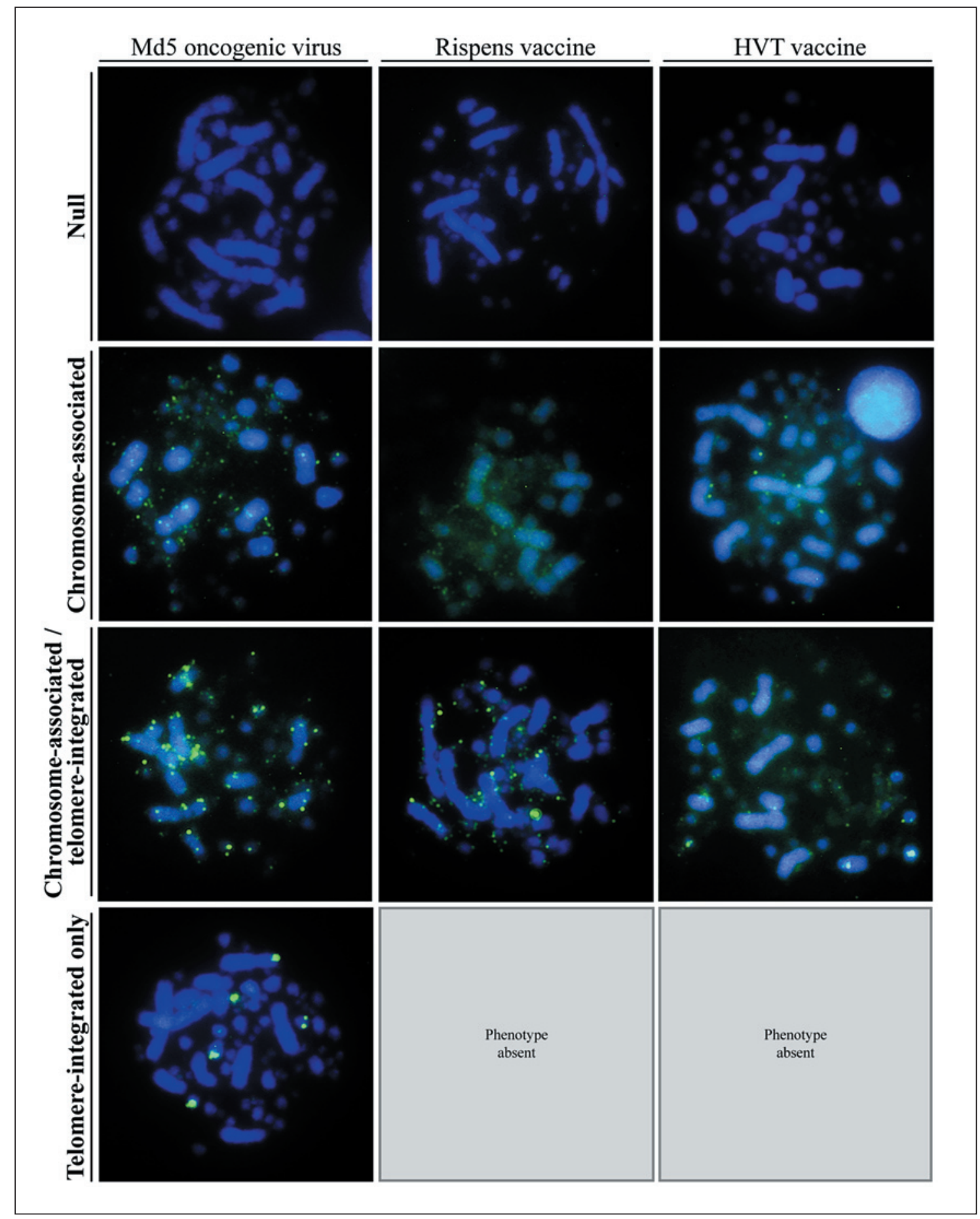

Fig. 1. Representative cytogenomic phenotypes of oncogenic Marek's disease (MD) virus (Md5 strain) or MD vaccine (Rispens and HVT) interactions with the host genome at 21 days after challenge or vaccination. The FISH images signify the status of the herpesvirus with regard to the chicken host genome in mitotic splenic-derived cells of disease-susceptible birds. The null phenotype lacks viral FISH signals (FITC, green) around the chromo- somes (DAPI, blue). The viral chromosome-associated phenotype is defined by diffuse viral FISH signals surrounding the host chromosomes, while the chromosome-associated/telomere-integrated phenotype consists of both the diffuse associated signals and bright, punctate integrated-virus signals at the telomeres. The telomere-integrated only viral phenotype is exclusively composed of distinct, punctate FISH MD virus signals at the telomeres. 

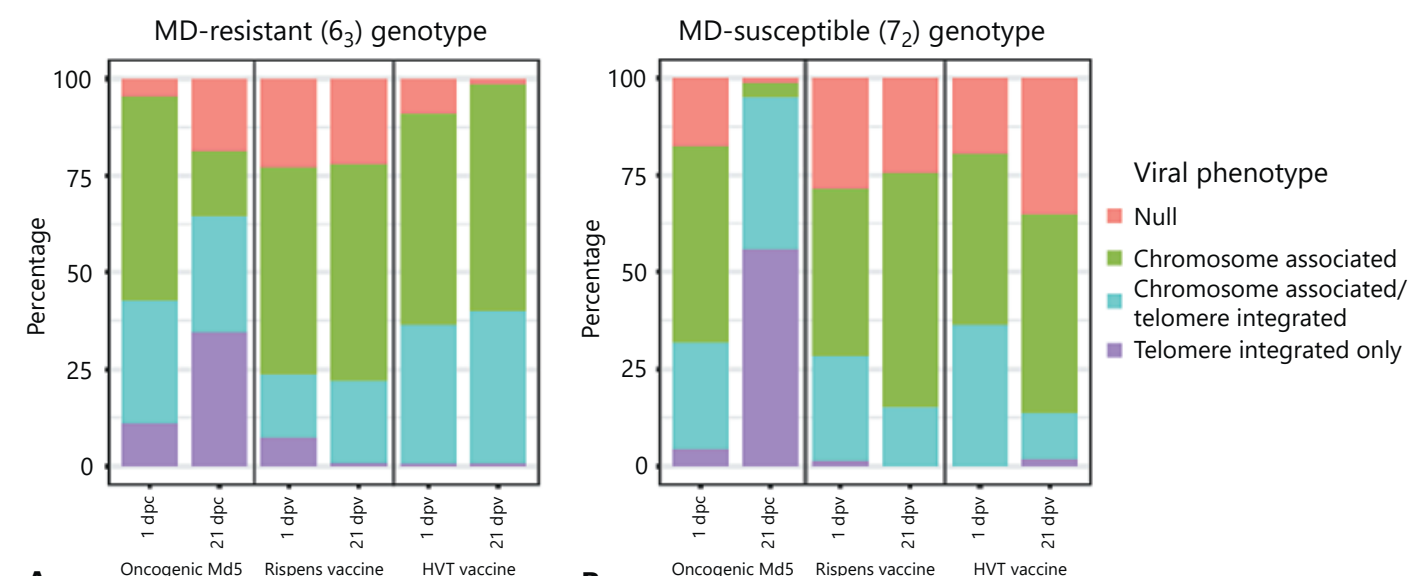

A

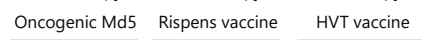

B

Oncogenic Md5 Rispens vaccine HVT vaccine

Fig. 2. Virus-host cytogenomic interactions following oncogenic Marek's disease (MD) virus (Md5 strain) or MD vaccine strain (Rispens or HVT) inoculation of disease resistant $\left(6_{3}\right)$ or susceptible $\left(7_{2}\right)$ genotypes. Each stacked bar graph represents the virushost cytogenomic phenotypes (see Fig. 1) observed for oncogenic MDV, the Rispens vaccine, or the HVT vaccine strain, respective- ly, at 1 day after challenge/vaccination (1-day-old birds) or 21 days after challenge/vaccination (3-week-old birds). The colored bars within each plot represent the mean percentages of mitotically dividing host cells with a given phenotype for 3 birds per treatment group. A Virus phenotypes for the $\sigma_{3}$ (MD-resistant) birds. B Virus phenotypes for the $7_{2}$ (MD-susceptible) birds.
Tukey's post-hoc test, as described above, to establish statistically significant differences within the genotypes (i.e., between the unvaccinated $+\mathrm{MDV}$-challenged and the vaccinated + MDV-challenged treatment groups). The effects of vaccination (treatment) and $\mathrm{dpc}$ (timepoint) on viral phenotype within each chicken genotype were calculated by this method. Statistically significant differences in the normalized viral phenotype data between the genotypes (for each timepoint) were, similarly, determined by multiple one-way ANOVA analyses, as described above.

\section{Results}

All cytogenomic data are represented in the figures and tables as average values from 3 individual splenicderived samples (3 different birds) per treatment type, timepoint, and genotype. The oncogenic and vaccine virus-host cell population phenotypes are shown in Figure 1 . The dynamic nature of the phenotypes over time (temporal) for the 2 genotypes and single or dual treatments over 1-21 days post treatments are found in online supplementary Figures 1 and 2 (for all online suppl. material, see www.karger.com/doi/10.1159/000495174), with all data comprehensively reported in online supplementary Tables 1 and 2. Here, we emphasize the key results for genotype and vaccine treatment virus/host interactions, as outlined in Figures 1-3.

\section{MD-Resistant Chicken Genotype $6_{3}$ : Comparisons of}

Oncogenic MDV and MD Vaccines at the Level of the

\section{Host Genome}

Notable features of the oncogenic MDV-host genomic interactions within MD-resistant birds were a significant increase in the viral telomere-integrated only phenotype concomitant with a decline in chromosome-associated phenotype by $21 \mathrm{dpc}$ (Fig. 2A). The nonlinear changes of the oncogenic MDV viral phenotype across timepoints indicated a variable or sporadic nature of oncogenic MDV genomic interactions during early infection in the MD-resistant birds (online suppl. Fig. 1).

The cytogenomic interactions of the MD vaccines with the host genome in the MD-resistant genotype significantly contrasted with those of oncogenic MDV at multiple points (online suppl. Table 1). The vaccine treatment groups almost completely lacked the telomere-integrated only phenotype across almost all experimental timepoints; as a result, this viral phenotype appeared in a significantly lower percentage of dividing cells as compared to the oncogenic MDV group from $7 \mathrm{dpc} / \mathrm{dpv}$ onwards. The Rispens-vaccinated birds had a higher mean percentage of null phenotype (no virus) cells in the splenic-derived samples as compared to the MDV-challenged birds at all timepoints of early infection except at $4 \mathrm{dpc} / \mathrm{dpv}$. The HVTvaccinated group showed a significant phenotypic pattern 


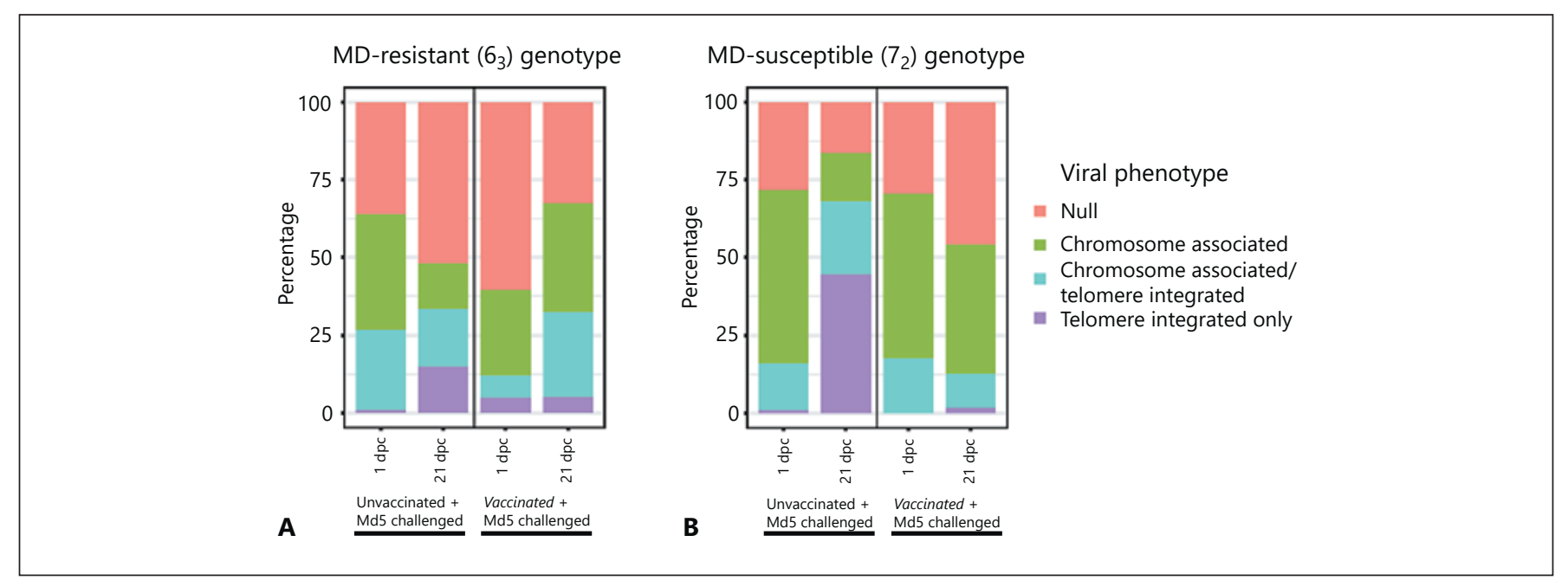

Fig. 3. Virus-host cytogenomic interactions of the oncogenic Marek's disease (MD) virus (Md5 strain) and/or the Rispens MD vaccine strain in unvaccinated + challenged birds or vaccinated + challenged birds of the $6_{3}$ disease resistant $(\mathbf{A})$ and $7_{2}$ susceptible (B) genotypes. Each stacked bar graph represents the virus-host cytogenomic phenotypes observed for oncogenic MDV and/or the

fluctuation at $7 \mathrm{dpv}$ with a sharp rise in the associated phenotype and dramatic decline in the "transitional" chromosome-associated/telomere-integrated phenotype (online suppl. Fig. 1). The MDV-challenged group showed a lower mean percentage of cells with the chromosome-associated viral phenotype than both MD vaccinated groups at 4 and $14 \mathrm{dpc} / \mathrm{dpv}$ and a significantly lower percentage as compared to the vaccine groups at $21 \mathrm{dpc} / \mathrm{dpv}$.

MD-Susceptible Chicken Genotype 72: Comparisons of Oncogenic MDV and the MD Vaccines at the Level of the Host Genome

In the MD lymphoma-susceptible genotype $7_{2}$ birds, there was a significant increase in the viral telomere-integrated only phenotype and a decrease in chromosomeassociated phenotype between 1 and $21 \mathrm{dpc}$ for the oncogenic MDV-challenged group (Fig. 2B), similar to the observation within genotype $6_{3}$. These phenotypic changes over the course of early infection were near linear, indicating a more predictable pattern of oncogenic MDVhost genomic interactions in MD-susceptible birds (online suppl. Fig. 1). Additionally, the mean percentage of cells with the MDV null phenotype showed a sharp decline at $21 \mathrm{dpc}$ (around the timing of lymphoma development), which was significantly less compared to all previous timepoints.

Vaccination and Host Genetics Impact MDV-Host Genome Interaction Profiles
Rispens vaccine strain in unvaccinated + challenged birds and Rispens-vaccinated (on day of hatch) + challenged birds, respectively, at 1 day after MDV challenge (5-day-old birds) or 21 days after MDV challenge (25-day-old birds). The colored bars within each plot represent the mean percentages of mitotically dividing host cells with a given phenotype for 3 birds per treatment group.

The patterns of chromosome association and integration for the MD vaccine-viruses within the MD-susceptible birds sharply contrasted with that of oncogenic MDV (Fig. 2B). The MD vaccine treatment groups contained almost no telomere-integrated only viral phenotype mitotic cells. The mean percentage for the integrated only phenotype was significantly less in the vaccinated groups versus the oncogenic MDV challenge group from 7 to 21 $\mathrm{dpc} / \mathrm{dpv}$ (online suppl. Table 2). Notably, the chromosome-associated viral phenotype was nearly absent in the oncogenic MDV-challenged birds at 14 and $21 \mathrm{dpc}$ but remained in a high $(>50 \%)$ percentage of splenic cells in the vaccinated birds across both timepoints. At $21 \mathrm{dpc} /$ $\mathrm{dpv}$, the challenged group lacked a population of MDVfree cells, while the null (virus-free) phenotype persisted at significantly higher levels in the vaccinated birds (Fig. 2B). Unlike in the MD-resistant genotype, the HVT and Rispens vaccine strains did not reveal significant viral-phenotype pattern fluctuations between timepoints.

\section{Genotypes $6_{3}$ versus $7_{2}$ : Interactions between}

Oncogenic MDV Strain and the Host Genome

The oncogenic MDV-challenged birds from both genotypes showed a decline in the chromosome-associated viral phenotype and a rise in the telomere-integrated only phenotype within dividing cells during early infection (Fig. 2). 
From $7 \mathrm{dpc}$ to the later timepoints, the MD-resistant genotype had higher mean percentages of chromosome-associated phenotype cells ( $p \leq 0.01$ at 7 and $14 \mathrm{dpc}$ and $p \leq 0.05$ at $21 \mathrm{dpc}$ ) and lower mean percentages of telomere-integrated only phenotype cells $(p \leq 0.1$ at $7 \mathrm{dpc}$ and $p \leq 0.05$ at $14 \mathrm{dpc}$ ) (online suppl. Fig. 1). The mean percentage of MDV-free (null) splenic-derived cells was significantly higher in the MD-resistant birds by $21 \mathrm{dpc}$ (Fig. 2A).

\section{Genotypes $6_{3}$ versus $7_{2}$ : Interactions between Rispens} or HVT Vaccines and the Host Genome

The Rispens vaccination treatment groups for both chicken genotypes displayed similar patterns of MDV chromosome association and integration phenotypes in splenic-derived, mitotically dividing cells from 1 to 21 dpv (Fig. 2). In one exceptional instance, the MD-resistant birds indicated a higher percentage of MDV-free (null phenotype) cells as compared to the susceptible chicken genotype at 2 weeks after vaccination $(p \leq 0.01)$ (online suppl. Fig. 1). Furthermore, the telomere-integrated only phenotype was not detected in splenic-derived cells of the Rispens-vaccinated MD-resistant and -susceptible birds, with a few exceptional incidences (online suppl. Tables 1,2).

The HVT-vaccinated treatment groups had inversely fluctuating mean percentages of viral chromosome-associated phenotype and viral associated/integrated phenotype cells in both genotypes (online suppl. Fig. 1). The difference in the mean values for these viral phenotypes between the genotypes was significant at 4 and $7 \mathrm{dpv}$. These fluctuations indicate a highly variable nature of HVT-host genome interactions in the dividing cell population. The difference in the associated/integrated HVT phenotype between the genotypes was statistically significant at $21 \mathrm{dpv}$. The high percentage of cells with the associated/integrated viral phenotype in the resistant birds and the large decline of this phenotype in the susceptible birds at $21 \mathrm{dpv}$ was accompanied by a respective decrease and increase in the percentage of HVT-free (null phenotype) dividing cells (Fig. 2). Similar to the Rispens-vaccinated group, the HVT-vaccinated group did not develop a population of telomere-integrated only splenic-derived cells at any timepoints, with a few cells as rare exceptions (online suppl. Tables 1, 2).

\section{MD-Resistant Genotype $6_{3}$ : Comparisons of}

Unvaccinated and Vaccinated Birds after Challenge

with Oncogenic MDV

The MDV-host genomic interactions within MD-resistant birds were altered by day-of-hatch vaccination with Rispens, determined by comparison to unvaccinated and MDV-challenged resistant birds (online suppl. Fig. 2). At later timepoints ( 14 and $21 \mathrm{dpc}$ ), the population of splenic-derived cells with the viral telomere-integrated only phenotype was higher in unvaccinated birds. These cells represent solely an MDV infection (i.e., no vaccine present). At $1 \mathrm{dpc}$, the vaccinated resistant birds, versus unvaccinated, showed a significantly higher population of splenic-derived dividing cells free of virus (null phenotype) concomitant with a lower population of viral chromosome-associated/telomere-integrated phenotype cells (Fig. 3A). At 21 days after MDV challenge, the vaccinated birds indicated significantly more viral chromosome-associated phenotype in splenic-derived cells. Aside from these instances, the percentages of the host mitotic cell populations with each virus-host phenotype were not significantly different between the vaccinated and unvaccinated birds (online suppl. Fig. 2).

\section{MD-Susceptible Genotype 72: Comparisons of}

\section{Unvaccinated and Vaccinated Birds after Challenge}

\section{with Oncogenic MDV}

The cytogenomic interactions of MDV with the host genome in the MD-susceptible genotype greatly contrasted between the Rispens-vaccinated and unvaccinated groups (Fig. 3B). The vaccinated and challenged treatment group almost completely lacked the MDV telomereintegrated only phenotype across all of the experimental timepoints (online suppl. Table 3). As a result, this viral phenotype appeared in a significantly lower percentage of cells as compared to the unvaccinated group from $4 \mathrm{dpc}$ onwards (online suppl. Fig. 2). Importantly, this phenotype represents solely MDV infection of the dividing host cell. The Rispens-vaccinated/MDV-challenged group had a higher mean percentage of viral null phenotype (no virus) and chromosome-associated (replicating virus) phenotype cells in the spleen as compared to the unvaccinated group. This higher percentage was significant for the null phenotype from 7 through 21 days post MDV challenge and for the associated phenotype at $21 \mathrm{dpc}$. The percentage of host cells with the transitional, chromosome-associated/telomere-integrated phenotype was generally similar between the unvaccinated and vaccinated susceptible birds, except for at 7 days post MDV challenge.

Genotypes $6_{3}$ versus $7_{2}$ : Genotype Effects on Virus-

Host Genomic Interactions in Rispens-Vaccinated and

Oncogenic MDV-Challenged Birds

The unvaccinated disease-resistant versus -susceptible genotype birds greatly differed in their virus-host cytoge- 
nomic interactions after challenge with oncogenic MDV at $4 \mathrm{dph}$ (online suppl. Fig. 2), as was observed for the birds challenged with MDV at hatch (online suppl. Fig. 1). However, day-of-hatch vaccination for MD, prior to challenge, altered the virus-host interactions to a similar outcome in disease-susceptible and -resistant birds (Fig. 3). Rispens-vaccinated/MDV-challenged (i.e., dual treatment) resistant $\left(6_{3}\right)$ and susceptible $\left(7_{2}\right)$ birds indicated analogous patterns of viral chromosome association and integration phenotypes (online suppl. Fig. 2), and statistically significant differences between the genotypes were scarce (online suppl. Table 4). The resistant birds indicated a significantly lower percentage of viral chromosome-associated phenotype dividing cells at 1 and $4 \mathrm{dpc}$ as compared to the susceptible birds $(p \leq 0.05)$. The MD-resistant birds also indicated significantly more virus-free dividing cells at 1 and $4 \mathrm{dpc}$. Notably, the telomere-integrated only phenotype was rarely detected in the spleen mitotic cell populations of vaccinated/challenged birds from both genotypes (Fig. 3).

\section{Discussion}

MD in commercial chicken populations is largely controlled by selective breeding for viability and disease resistance coupled with widespread vaccination. Our understanding of the biological and molecular mechanisms behind these preventative measures and the interplay between MDV and the host genome, especially with regard to bird genotype, is limited. The inbred chicken genotypes $\sigma_{3}$ (MD resistant) and $7_{2}$ (MD susceptible) share the same MHC haplotype, yet have unique genetic backgrounds and immune responses that contribute to their dissimilarity in MD susceptibility [Palladino et al., 1977; Lee et al., 1981; Vallejo et al., 1997; Baigent et al., 1998; Kaiser et al., 2003; Zhang et al., 2006]. Our cytogenomic results provide new insights with regard to host genetic background effects on pathogenic MDV and MD vaccine-virus behavior, encompassing viral replication, integration, and latency, with single-cell resolution. Our data also outline the effect of Rispens vaccination on oncogenic MDV interactions with the host genome in resistant and susceptible birds, as compared to the interactions observed in unvaccinated birds. The viral infection stages represented by the virus-host phenotypes in splenic-derived, mitotically dividing cells were previously described [Robinson et al., 2014; McPherson and Delany, 2016; McPherson et al., 2016], and this work confirms that MD vaccines can integrate into the host genome, but do not

Vaccination and Host Genetics Impact MDV-Host Genome Interaction Profiles generate a robust telomere-integrated only cell population as seen with oncogenic viruses [McPherson et al., 2016]. The exceptionally rare instances of the Rispens integrated-only viral phenotype may represent transformation-susceptible host cells induced by MD vaccine virus. Furthermore, the cytogenomic profiles for all $3 \mathrm{MDV}$ strains indicated MDV-positive dividing host cells at surprisingly high levels during early timepoints after challenge or vaccination ( 1 and $4 \mathrm{dpv} / \mathrm{dpc}$ ). This finding indicates that oncogenic MDV and MD vaccines may preferentially infect replicating host lymphocytes and/or induce cellular proliferation upon initial infection, thus resulting in elevated representation within mitotic cellspecific datasets.

For both chicken genotypes in this study, oncogenic MDV showed a decline in viral replication and significant emergence of latent virus, as represented by the telomereintegrated phenotypes, around the timing of MD tumor development (21 dpc). The MD vaccines, which lack oncogenic transformation potential, conserved the viral replication stage later into the infection (through $21 \mathrm{dpv}$ ). These results indicate that vulnerability to development of MD lymphomas, or the oncogenic potential of an MDV infection, is correlated with an elevated dividing cell population with telomere-integrated oncogenic MDV and a loss of the virus-free cells in the lymphoid organs. The reemergence of Rispens and HVT vaccines viral replication may stem from the absence of the "classic" switch of pathogenic MDV to the latency and oncogenic transformation herpesvirus stages. In contrast, some studies have indicated a positive correlation between MDV virulence and replication levels in host cells [Yunis et al., 2004; Dunn et al., 2014]. The contrast in our findings could be explained by a less productive vaccinevirus replication (lower viral genome production detected by quantitative PCR methods) occurring in a large population of dividing splenic lymphocytes within the vaccinated host [McPherson et al., 2016].

The improved clearing of highly oncogenic MDV in the MD-resistant $\sigma_{3}$ birds seems unsurprising, as the reduced presence of pathogenic viral genomes in host cells would decrease the probability of disease progression and tumor development. The greater presence of the telomere-integrated only phenotype in MD-susceptible hosts supports a strong correlation between this MDV phenotype and MD-induced oncogenic transformation and tumor cell populations, as seen in prior studies [Robinson et al., 2010, 2014; McPherson et al., 2016]. This correlation may be underlined by increased proliferation of transformed cells with integrated virus, thereby increas- 
ing the proportion of MDV-integrated phenotype cells in lymphoid tissue(s). Integrated-only phenotype mitotic cells were also detected (at significantly lower levels) in challenged MD-resistant hosts, suggesting that the profusion of this viral phenotype, rather than its presence alone, in the lymphoid tissue is the strongest indicator of MD tumor development susceptibility. As a whole, these cytogenomic findings indicated unique early-infection patterns of virus-host phenotypes for pathogenic MDV and the apathogenic HVT and Rispens MD vaccines.

Our investigations of vaccination and subsequent MDV challenge in the same host bird genotypes (i.e., dual-treatment group) provided insights on the impact of vaccination on oncogenic MDV behavior. Analyses of immune cell populations in vaccinated/challenged host birds, in the absence of differential labeling between vaccine and oncogenic MDV strains, were possible due to the scarcity of the telomere-integrated only phenotype in Rispens-vaccinated only birds. Vaccination with Rispens significantly reduced the percentage of splenic-derived dividing cells with the telomere-integrated only phenotype and resulted in more virus-free cells after MDV challenge in disease-susceptible hosts. The disease-resistant genotype demonstrated less contrast in vaccinated versus unvaccinated outcomes, likely because of the genotypebased resistance to MD tumor development in these birds. The larger population of chromosome-associated phenotype cells in the vaccinated birds aligns with our knowledge that vaccination for MD does not induce sterilizing immunity and, thus, host immune cells may contain replicating vaccine and/or oncogenic MDV without inducing disease symptoms. The low presence of the telomere-integrated only phenotype in the vaccinated (i.e., protected) birds may be a hallmark of effective vaccination against MD and, further, supports the hypothesis that this viral integration phenotype represents a cell primed for virus-induced transformation. Through use of single-cell cytogenomic approaches for evaluation of viral dynamics, as described here, the MDV telomere integrated-only phenotype abundance in lymphoid tissues may prove to be a strong candidate for evaluating MD vaccine protective efficacy. Furthermore, the signature virus-host dynamics involved in vaccinal protection, disease progression, and MD-induced oncogenesis, established by this study, provide analytical tools in the effort to continuously protect the world's poultry populations from serious MD outbreaks.

\section{Acknowledgements}

This project was supported by Agriculture and Food Research Initiative Competitive Grant nos. 2013-67015-21330 and 200535205-16679 from the USDA National Institute of Food and Agriculture, National Research Support Program-8 (CA-D*-ASC7233-RR), and Multi-State Research Projects NC-1170 (CAD*-ASC-6414-RR) and NE-1334 (CA-D*-ASC-7281-RR). The authors acknowledge the infrastructure support of the Department of Animal Science and the College of Agricultural and Environmental Sciences and the California Agricultural Experiment Station at the University of California, Davis. We greatly appreciate the research contributions of Laurie Molitor at ADOL.

\section{Statement of Ethics}

The authors declare that all experiments performed in this study comply with the current laws of the United States of America. All animal experiments were carried out in accordance with the National Institutes of Health guide for the care and use of Laboratory animals (NIH Publications No. 8023, revised 1978) and the guidelines set forth by the USDA, ARS, ADOL Institutional Animal Care and Use Committee (IACUC).

\section{Disclosure Statement}

The authors have no conflicts of interest to declare.

\section{References}

Adldinger H, Calnek B: Pathogenesis of Marek's disease: early distribution of virus and viral antigens in infected chickens. J Natl Cancer Inst 50:1287-1298 (1973).

Arumugaswami V, Kumar PM, Konjufca V, Dienglewicz RL, Reddy SM, Parcells MS: Latency of Marek's disease virus (MDV) in a reticuloendotheliosis virus-transformed T-cell line. II. Expression of the latent MDV genome. Avian Dis 53:156-165 (2009).
Bacon LD: Influence of the major histocompatibility complex on disease resistance and productivity. Poult Sci 66:802-811 (1987).

Bacon LD, Witter RL: Influence of turkey herpesvirus vaccination on the $\mathrm{B}$-haplotype effect on Marek's disease resistance in 15.B-congenic chickens. Avian Dis 36:378-385 (1992).

Bacon LD, Witter RL: Influence of B-haplotype on the relative efficacy of Marek's disease vaccines of different serotypes. Avian Dis 37:5359 (1993).
Bacon LD, Witter RL: Serotype specificity of Bhaplotype influence on the relative efficacy of Marek's disease vaccines. Avian Dis 38:65-71 (1994).

Bacon LD, Hunt HD, Cheng HH: A review of the development of chicken lines to resolve genes determining resistance to diseases. Poult Sci 79:1082-1093 (2000). 
Baigent SJ, Davison F: Marek's disease virus: biology and life cycle, in Davison F, Nair V (eds): Marek's Disease, an Evolving Problem, pp 62-77 (Elsevier Academic Press, Amsterdam 2004).

Baigent SJ, Ross LJ, Davison TF: Differential susceptibility to Marek's disease is associated with differences in number, but not phenotype or location, of pp38+ lymphocytes. J Gen Virol 79:2795-2802 (1998).

Baigent SJ, Smith LP, Currie RJW, Nair VK: Replication kinetics of Marek's disease vaccine virus in feathers and lymphoid tissues using PCR and virus isolation. J Gen Virol 86:29892998 (2005).

Biggs PM: Marek's disease - current state of knowledge, in Arber W, Braun W, Cramer F, Haas R, Henle W, et al (eds): Current Topics in Microbiology and Immunology, vol 43, pp 92-125 (Springer, Heidelberg 1968).

Briles WE, Briles RW, Taffs RE, Stone HA: Resistance to a malignant lymphoma in chickens is mapped to subregion of major histocompatibility (B) complex. Science 219:977-979 (1983).

Burgess SC, Davison TF: Identification of the neoplastically transformed cells in Marek's disease herpesvirus-induced lymphomas: recognition by the monoclonal antibody AV37. J Virol 76:7276-7292 (2002).

Calnek BW: Pathogenesis of Marek's disease virus infection, in Hirai K (ed): Marek's Disease, pp 25-55 (Springer, Heidelberg 2001).

Calnek BW, Witter RL: Marek's disease virus, in Calnek BW, Barnes HJ, Beard CW, McDougald LR, Saif YM (eds): Disease of Poultry, ed 9, pp 369-413 (Iowa State University Press, Ames 1997).

Calnek BW, Schat KA, Peckham MC, Fabricant J: Field trials with a bivalent vaccine (HVT and SB-1) against Marek's disease. Avian Dis 27: 844-849 (1983).

Chang S, Dunn JR, Heidari M, Lee LF, Song J, et al: Genetics and vaccine efficacy: host genetic variation affecting Marek's disease vaccine efficacy in White Leghorn chickens. Poult Sci 89:2083-2091 (2010)

Chang S, Dunn JR, Heidari M, Lee LF, Ernst CW, et al: Vaccine by chicken line interaction alters the protective efficacy against challenge with a very virulent plus strain of Marek's disease virus in White Leghorn chickens. World J Vaccines 2:1-11 (2012)

Chang S, Xie Q, Dunn JR, Ernst CW, Song J, Zhang H: Host genetic resistance to Marek's disease sustains protective efficacy of herpesvirus of turkey in both experimental and commercial lines of chickens. Vaccine 32:18201827 (2014).

Cheng HH, Perumbakkam S, Pyrkosz AB, Dunn JR, Legarra A, Muir WM: Fine mapping of QTL and genomic prediction using allele-specific expression SNPs demonstrates that the complex trait of genetic resistance to Marek's disease is predominantly determined by transcriptional regulation. BMC Genomics 16: 816 (2015)
Churchill AE, Biggs PM: Agent of Marek's disease in tissue culture. Nature 215:528-530 (1967).

Churchill AE, Biggs PM: Herpes-type virus isolated in cell culture from tumors of chickens with Marek's disease. II. Studies in vivo. J Natl Cancer Inst 41:951-956 (1968).

Churchill AE, Payne LN, Chubb RC: Immunization against Marek's disease using a live attenuated virus. Nature 221:744-747 (1969).

Delany ME, Gessaro TM, Rodrigue KL, Daniels LM: Chromosomal mapping of chicken mega-telomere arrays to GGA9, 16, 28 and W using a cytogenomic approach. Cytogenet Genome Res 117:54-63 (2007).

Delecluse HJ, Hammerschmidt W: Status of Marek's disease virus in established lymphoma cell lines: herpesvirus integration is common. J Virol 67:82-92 (1993).

Dunn JR, Auten K, Heidari M, Buscaglia C: Correlation between Marek's disease virus pathotype and replication. Avian Dis 58:287-292 (2014).

Gimeno IM, Witter RL, Cortes AL, Reed WM: Replication ability of three highly protective Marek's disease vaccines: implications in lymphoid organ atrophy and protection. Avian Pathol 40:573-579 (2011).

Greco A, Fester N, Engel AT, Kaufer BB: Role of the short telomeric repeat region in Marek's disease virus replication, genomic integration, and lymphomagenesis. J Virol 88: 14138-14147 (2014).

Haq K, Brisbin JT, Thanthrige-Don N, Heidari M, Sharif S: Transcriptome and proteome profiling of host responses to Marek's disease virus in chickens. Vet Immunol Immunopathol 138:292-302 (2010).

Haq K, Fear T, Ibraheem A, Abdul-Careem MF, Sharif S: Influence of vaccination with CVI988/Rispens on load and replication of a very virulent Marek's disease virus strain in feathers of chickens. Avian Pathol 41:69-75 (2012).

Islam AFMF, Walkden-Brown SW, Groves PJ, Underwood GJ: Effects of vaccine dose, virus challenge dose and interval from vaccination to challenge on protection of broiler chickens against Marek's disease virus challenge. Aust Vet J 85:348-355 (2007).

Islam T, Renz KG, Walkden-Brown SW, Ralapanawe S: Viral kinetics, shedding profile, and transmission of serotype 1 Marek's disease vaccine Rispens/CVI988 in maternal antibody-free chickens. Avian Dis 57:454-463 (2013).

Kaiser P, Underwood G, Davison F: Differential cytokine responses following Marek's disease virus infection of chickens differing in resistance to Marek's disease. J Virol 77:762-768 (2003).

Kaschka-Dierich C, Nazerian K, Thomssen R: Intracellular state of Marek's disease virus DNA in two tumour-derived chicken cell lines. J Gen Virol 44:271-280 (1979).
Kaufer BB, Jarosinski KW, Osterrieder N: Herpesvirus telomeric repeats facilitate genomic integration into host telomeres and mobilization of viral DNA during reactivation. J Exp Med 208:605-615 (2011).

Kingham BF, Zelník V, Kopácek J, Majerciak V, Ney E, Schmidt CJ: The genome of herpesvirus of turkeys: comparative analysis with Marek's disease viruses. J Gen Virol 82:11231135 (2001).

Lee LF, Powell PC, Rennie M, Ross LJ, Payne LN: Nature of genetic resistance to Marek's disease in chickens. J Natl Cancer Inst 66:789796 (1981).

McPherson MC, Delany ME: Virus and host genomic, molecular, and cellular interactions during Marek's disease pathogenesis and oncogenesis. Poult Sci 95:412-429 (2016).

McPherson MC, Cheng HH, Delany ME: Marek's disease herpesvirus vaccines integrate into chicken host chromosomes yet lack a virushost phenotype associated with oncogenic transformation. Vaccine 34:5554-5561 (2016).

Miller MM, Taylor RL: Brief review of the chicken major histocompatibility complex: the genes, their distribution on chromosome 16, and their contributions to disease resistance. Poult Sci 95:375-392 (2016).

Nair V: Evolution of Marek's disease - a paradigm for incessant race between the pathogen and the host. Vet J 170:175-183 (2005).

Okazaki W, Purchase HG, Burmester BR: Protection against Marek's disease by vaccination with a herpesvirus of turkeys. Avian Dis 14: 413-429 (1970).

Osterrieder N, Kamil JP, Schumacher D, Tischer BK, Trapp S: Marek's disease virus: from miasma to model. Nat Rev Microbiol 4:283-294 (2006).

Palladino MA, Gilmour DG, Scafuri AR, Stone HA, Thorbecke GJ: Immune response differences between two inbred chicken lines identical at the major histocompatibility complex. Immunogenetics 5:253-259 (1977).

Petherbridge L, Howes K, Baigent SJ, Sacco MA, Evans S, et al: Replication-competent bacterial artificial chromosomes of Marek's disease virus: novel tools for generation of molecularly defined herpesvirus vaccines. J Virol 77: 8712-8718 (2003).

$\mathrm{R}$ Development Core Team: R: a language and environment for statistical computing (The $\mathrm{R}$ Foundation for Statistical Computing, Vienna 2016). http://www.R-project.org/

Rispens BH, van Vloten H, Mastenbroek N, Maas HJ, Schat KA: Control of Marek's disease in the Netherlands. I. Isolation of an avirulent Marek's disease virus (strain CVI 988) and its use in laboratory vaccination trials. Avian Dis 16:108-125 (1972)

Robinson CM, Hunt HD, Cheng HH, Delany ME: Chromosomal integration of an avian oncogenic herpesvirus reveals telomeric preferences and evidence for lymphoma clonality. Herpesviridae 1:5 (2010). 
Robinson CM, Cheng HH, Delany ME: Temporal kinetics of Marek's disease herpesvirus: integration occurs early after infection in both $\mathrm{B}$ and T cells. Cytogenet Genome Res 144:142154 (2014).

Sarson AJ, Parvizi P, Lepp D, Quinton M, Sharif S: Transcriptional analysis of host responses to Marek's disease virus infection in genetically resistant and susceptible chickens. Anim Genet 39:232-240 (2008).

Schat KA, Calnek BW: Characterization of an apparently nononcogenic Marek's disease virus. J Natl Cancer Inst 60:1075-1082 (1978).

Schat KA, Taylor RL Jr, Briles WE: Resistance to Marek's disease in chickens with recombinant haplotypes of the major histocompatibility (B) complex. Poult Sci 73:502-508 (1994).

Sharma JM, Stone HA: Genetic resistance to Marek's disease. Delineation of the response of genetically resistant chickens to Marek's disease virus infection. Avian Dis 16:894-906 (1972).
Silva RF, Dunn JR, Cheng HH, Niikura M: A MEQ-deleted Marek's disease virus cloned as a bacterial artificial chromosome is a highly efficacious vaccine. Avian Dis 54:862-869 (2010).

Spatz SJ, Schat KA: Comparative genomic sequence analysis of the Marek's disease vaccine strain SB-1. Virus Genes 42:331-338 (2011).

Spatz SJ, Petherbridge L, Zhao Y, Nair V: Comparative full-length sequence analysis of oncogenic and vaccine (Rispens) strains of Marek's disease virus. J Gen Virol 88:10801096 (2007).

Stone HA: Use of highly inbred chickens in research. Agricultural Research Service, United States Department of Agriculture (1975). http://handle.nal.usda.gov/10113/ CAT75663574.

Tan J, Cooke J, Clarke N, Tannock GA: Molecular evaluation of responses to vaccination and challenge by Marek's disease viruses. Avian Pathol 36:351-359 (2007).
Trapp S, Parcells MS, Kamil JP, Schumacher D, Tischer BK, et al: A virus-encoded telomerase RNA promotes malignant $\mathrm{T}$ cell lymphomagenesis. J Exp Med 203:1307-1317 (2006).

Vallejo RL, Pharr GT, Liu HC, Cheng HH, Witter RL, Bacon LD: Non-association between $R f p-Y$ major histocompatibility complex-like genes and susceptibility to Marek's disease virus-induced tumours in $6_{3} \times 7_{2} \mathrm{~F} 2$ intercross chickens. Anim Genet 28:331-337 (1997).

Witter RL: A new strategy for Marek's disease immunisation - bivalent vaccine. Avian Pathol 13:133-135 (1984)

Yunis R, Jarosinski KW, Schat KA: Association between rate of viral genome replication and virulence of Marek's disease herpesvirus strains. Virology 328:142-150 (2004).

Zhang HM, Hunt HD, Kulkarni GB, Palmquist DE, Bacon LD: Lymphoid organ size varies among inbred lines $6_{3}$ and $7_{2}$ and their thirteen recombinant congenic strains of chickens with the same major histocompatibility complex. Poult Sci 85:844-853 (2006). 